\title{
Lorsque le droit nord-américain des sociétés dessine les nouvelles frontières de l'entreprise : les clés pour un autre futur?
}

\author{
par Ivan Tchotourian
}

\begin{abstract}
IVAN, TCHOTOURIAN
Maître de conférences (Université de Nantes), Docteur en droit privé, membre de l'I.R.D.P. (Université de Nantes) et du C.R.D.F. (Université Paris 1 Panthéon-Sorbonne), chercheur associé à la C.D.A.C.I. (Université de Montréal), ancien titulaire de la bourse de recherche Lavoisier (EGIDE)
\end{abstract}

15 , rue Gérando

75009 Paris

Université de Nantes

3, rue du Maréchal Joffre

BP 34103

44041 NANTES Cedex 1

Tél. : + 33 (0)2.40.30.60.23

Courriel : ivan.tchotourian@univ-nantes.fr 


\title{
Lorsque le droit nord-américain des sociétés dessine les nouvelles frontières de l'entreprise : les clés pour un autre futur?
}

\author{
par Ivan Tchotourian
}

IVAN, TCHOTOURIAN

Maître de conférences (Université de Nantes), Docteur en droit privé, membre de l'I.R.D.P. (Université de Nantes) et du C.R.D.F. (Université Paris 1 Panthéon-Sorbonne), chercheur associé à la C.D.A.C.I. (Université de Montréal), ancien titulaire de la bourse de recherche Lavoisier (EGIDE)

\section{Résumé}

Lorsque le droit nord-américain des sociétés dessine les nouvelles frontières de l'entreprise : les clés pour un autre futur?

par Ivan Tchotourian

Alors que le droit nord-américain des sociétés a longtemps adopté une lecture restrictive de l'entreprise en faisant de l'actionnaire son maillon essentiel, le paysage juridique subi à l'heure actuelle de profondes mutations. En dépit d'une intensité différente, l'entreprise canadienne et l'entreprise américaine s'ouvrent, sous l'influence combinée de la jurisprudence et de la doctrine (qui ne font que rejoindre l'histoire), de plus en plus à leur environnement et font une place grandissante au développement durable, à la responsabilité sociale des entreprises et à la stakeholder theory. Cette étude démontre ad fine le rôle essentiel qu'est amené à jouer l'idée de collectivité. Aussi positives que soient les conséquences attachées à cette observation en termes de construction d'un futur viable dans le long terme, celle-ci est source de questionnements et impose de repenser non seulement le dessin des nouvelles frontières de l'entreprise, mais encore la signification du concept de performance. Amérique du Nord, Entreprise, Droit des sociétés, Evolution, Perception nouvelle

\section{Abstract}

When corporate law proposes new borders for companies : the keys for another future? by Ivan Tchotourian

While the North American corporate law adopted for a long time a restrictive reading of the company by making of the shareholder its essential link, profound transformations affect the legal landscape. In spite of a different intensity, the Canadian company and the American company open, under the influence by the case law and by the doctrine (which are joining the history), more and more to their environment and make a growing place to the sustainable development, the corporate social responsibility and the stakeholder theory. This study 
demonstrates ad fine the essential role of the idea of community. However positive are the consequences attached to this observation in terms of construction of viable future in the long term, this one is source of questionings and imposes to rethink the drawing of the borders of the company and the meaning of the concept of performance.

North-America, Company, Corporate law, Evolution, Innovative definition.

\section{Resumen}

Cuando el derecho norteamericano de sociedades dibuja los nuevos límites de la empresa : la llave para otro futuro ?

Por Ivan Tchotourian

Mientras que el derecho norteamericano de sociedades adoptó durante mucho tiempo una interpretación restrictiva de la empresa haciendo del accionista su eslabón esencial, el paisaje jurídico de la actualidad sufre mutaciones profundas. A pesar de su diferencia en intensidad, la empresa canadiense y la empresa americana se abren, bajo la influencia combinada de la jurisprudencia y de la doctrina (que sólo relatan la historia), cada vez más a su entorno y abren paso de manera creciente, al desarrollo sostenible, a la responsabilidad social de las empresas y al Stakeholder Theory. Este estudio conlleva a demostrar el rol esencial que juega el concepto de colectividad. Por muy positivas que sean las consecuencias ligadas a esta observación en términos de construcción de un futuro viable a largo plazo, ésta es fuente de cuestionamientos e impone la necesidad de repensar no sólo los nuevos límites de la empresa, sino aun el concepto de perfeccionamiento.

América del Norte, empresa, derecho de sociedades, Evolución, nuevas perspectivas 


\section{Lorsque le droit nord-américain des sociétés dessine les nouvelles frontières de l'entreprise : les clés pour un autre futur ?*}

Apparue à la fin du $\mathrm{XX}^{\text {ème }}$ siècle et portée par la mondialisation, l'entreprise demeure une idée neuve à réinventer comme le met en exergue la tempête financière qui balaie le monde (F. Torres). Alors que la perception nord-américaine de l'entreprise couramment adoptée est construite autour d'un modèle essentiellement actionnarial qui trouve ses bases sur une relation d'agence et fait ergo propter hoc de l'entreprise un contrat (G. Charreaux (a) ; G. Charreaux (b) ; M. C. Jensen and W. H. Meckling ; A. A. Alchian and H. Demsetz), le droit change de physionomie et fait progressivement la place à une perception plurale de l'entreprise.

L'actionnaire n'est plus seul dans l'entreprise. Au regard de la structure molle et volatile qu'est devenue l'entreprise (B. Brunhes), de nouveaux acteurs, engagés contractuellement avec elle, que la nouvelle vague de dirigeants est loin d'ignorer sont apparus (A. IonescuSomers; A. Nick). Les parties prenantes - ou stakeholders - sont devenus des acteurs nécessaires à la création de valeur sans le support desquels l'organisation cesserait d'exister (J. Maati). Malgré des définitions théoriques distinctes, le développement durable, la responsabilité sociale et la stakeholder theory témoignent communément de l'existence d'une interface entre l'entreprise, ses acteurs et son environnement (J. Pasquero). Ces notions impliquent une prise en compte par l'entreprise des données économiques, environnementales et sociales (J. Roselle) et suppose que cette dernière s'ouvre à un horizon ne se limitant pas à celui des actionnaires (M. Capron et F. Quairel-Lanoizelée (a); M. Bonnafous-Boucher et Y. Pesqueux ; J. Roselle ; M. Capron et F. Quairel-Lanoizelée (b)).

Se réconciliant avec un passé pas si lointain qui n'hésitait pas à qualifier l'entreprise de «réalité sociale» reflétant un carrefour d'intérêts de force et de nature très divers (J.

\footnotetext{
* Une partie de cet article a été présentée lors du colloque international du 6 décembre 2007 consacré au thème "Prospective et entreprise », organisé conjointement par l'Université Paris Dauphine et l'IAE de Caen. Tout commentaire et suggestion relativement à ce papier seront les bienvenus et sont à envoyer à l'adresse suivante : ivan.tchotourian@univ-nantes.fr.
} 
Paillusseau. Egalement: M. Despax ; G. Ripert), le droit contemporain des sociétés par actions accorde en France une place grandissante aux stakeholders ... répondant en cela aux disciplines de la gestion, du mangement ou encore, de la stratégie, qui consacrent de nombreuses analyses à la théorie des parties prenantes (récemment : R. E. Freeman and $a l$.). Or, de manière identique à la France (I. Tchotourian $(b))$, l'activité législative canadienne en matière de sociétés par actions témoigne que les dirigeants de sociétés doivent de plus en plus tenir compte d'autres partenaires que les actionnaires (S. Rousseau). Si l'évolution de la construction règlementaire états-unienne paraît davantage en retrait, ce serait négliger non seulement les décisions judiciaires intervenues depuis l'arrêt de 1919 Dodge v. Ford Moto Co., mais encore l'opinion d'une partie des auteurs américains qui défend ouvertement une redéfinition du droit des sociétés dans un sens pro-stakeholder ou, qui se montrent à tout le moins réservés sur l'analyse contractuelle de la firme.

L'hypothèse de cet écrit est de vérifier que le paysage juridique nord-américain rend l'entreprise interactive à son environnement au travers de la place accordée aujourd'hui à la responsabilité sociale des entreprises (RSE), au développement durable ou encore, à la stakeholder theory (1. et 2.) et d'en présenter les conséquences eu égard à ses frontières qui semblent plus que jamais s'entourer d'un épais brouillard (3.).

Propos liminaires sur les situations européennes et françaises : A côté de l'Europe qui accorde une place indiscutable au développement durable et à la RSE, force est de constater que la France donne en parallèle forme à ce concept.

D'un côté, une multitude d'initiatives européennes en matière de développement durable témoigne de l'importance de la problématique RSE (par exemple : J. M., Lozano, L. Albareda and T. Ysa ; I. Tchotourian $(a))^{1}$. Il suffit de signaler les communications de la Commission européenne sur le développement durable qui se sont succédées depuis la communication du 2 juillet 2002 concernant «La responsabilité sociale des entreprises : une contribution des entreprises au développement durable » (communications du 23 décembre 2003, du 9 février 2005, du 23 décembre 2005 ou encore, du 22 mars 2006) ou la directive n²004-35 du 21

\footnotetext{
${ }^{1}$ Alors qu'aucune disposition du traité de Rome n'accordait de façon explicite compétence à la Communauté pour s'occuper de l'environnement, l'Europe a évolué à la fin de ce millénaire. Une décision des chefs d'Etat et de gouvernement en date du 20 octobre 1972 a invité les organes communautaires à se lancer dans une politique de l'environnement et une série de programmes quinquennaux d'action pour l'environnement a été adopté ainsi qu'une centaine d'instruments juridiques (le Conseil s'est appuyé sur les articles 100 et 235 du Traité). En 1986, la C.E.E. a adopté l'Acte unique qui a adapté le Programme des Nations Unies pour l'environnement. Des nouveaux articles $130 \mathrm{R}$ à $130 \mathrm{~T}$ ont défini les principes et les méthodes de l'action communautaire dans le domaine de l'environnement et une procédure spéciale a été prévue pour mettre en place de nouveaux instruments juridiques.
} 
avril 2004 instituant une responsabilité environnementale. A côté de ces positions du droit communautaire, l'importance du développement durable se reflète dans le Traité instituant la Communauté Européenne du 25 mars 1957 (article 2, article 3 notamment le point j, article 174 Titre XIX, article 177 Titre XX), dans le Traité sur l'Union européenne signé à Maastricht le 7 février 1992 (Préambule, article 2) et ressort également du projet de Constitution européenne. Par ailleurs, les organes décisionnels de l’Union européenne apportent une contribution certaine à la construction d'un cadre à la RSE comme en atteste les positions du Conseil européen du 16 et 17 juin 2005 et du Parlement européen du 13 mars 2007. Enfin, des organes non décisionnels de l'Union européenne contribuent à la construction et à l'appréhension du développement durable. Le Conseil européen n'a-t-il pas fait du développement durable un objectif clé dans sa déclaration sur les principes directeurs du développement durable de juin 2005 ?

D'un autre côté, la France intègre dans son corpus règlementaire de nouveaux paradigmes qui remettent aussi en cause les frontières de l'entreprise (dernièrement: F.-X. Lucas ; F.-G. Trébulle $(a)$; F.-G. Trébulle $(b))$. Le droit français des sociétés s'ouvre à un nouvel horizon qui dépasse celui des actionnaires. En ce sens, la loi du 30 juillet 2003 relative à la prévention des risques technologiques et naturels et à la réparation des dommages a mis à la charge de toutes les sociétés anonymes une obligation d'information sur la « (...) politique de prévention du risque d'accident technologique menée par la société », la «(...) capacité de la société à couvrir sa responsabilité civile vis-à-vis des biens et des personnes du fait de l'exploitation de telles installations» et les «(...) moyens prévus par la société pour assurer la gestion de l'indemnisation des victimes en cas d'accident technologique engageant sa responsabilité » (article L. 225-102-2 du Code de commerce). En outre, depuis la loi du 15 mai 2001 relative aux nouvelles régulations économiques, un alinéa 4 à l'article L. 225-102-1 du Code de commerce impose aux dirigeants d'une société dont les titres sont admis aux négociations sur un marché réglementé d'indiquer dans leur rapport de gestion «(...) la manière dont la société prend en compte les conséquences sociales et environnementales de son activité ». Enfin, l'information sociale et environnementale est devenue une inscription comptable au travers de la transposition de la directive du 18 juin 2003. Cette modification législative a renforcé le contenu du rapport de gestion d'un certain nombre de sociétés par actions dont les sociétés anonymes, les sociétés à responsabilité limitée et les sociétés en commandite par actions. Dorénavant, ce rapport (qui est communiquée aux actionnaires) doit comprendre une analyse des indicateurs clés de performance de nature non financière ayant trait à l'activité spécifique de la société, notamment des informations relatives aux questions d'environnement 
et de personnel avec, le cas échéant, des renvois aux montants indiqués dans les comptes annuels et des explications supplémentaires (article L. 225-100 alinéas 3, 4 et 5 du Code de commerce).

\section{Etat des lieux en droit canadien : les bouleversements jurisprudentiels actuels comme écho à l'histoire}

$\mathrm{Si}$, en droit canadien, l'entreprise a longtemps été perçue comme une institution noyant le conflit social opposant les seuls employés aux dirigeants (M. Martel et P. Martel), celle-ci est redéfinie aujourd'hui comme un lieu de conflit potentiel institutionnalisé entre différents acteurs. L'histoire (1.1.) et la jurisprudence actuelle (1.2.) se combinent pour démontrer que l'entreprise échappe de plus en plus à la conception nord-américaine qui ne voit en elle qu'un amalgame d'actionnaires.

\subsection{Histoire du droit canadien des sociétés : des frontières de moins en moins perceptibles}

L'évolution historique de la réglementation autorise l'élargissement de la société et, de son intérêt, à d'autres que les actionnaires. Une des premières évolutions marquantes est l'Acte pour fournir de l'eau à la Cité de Montréal et aux parties adjacentes adoptée par l'assemblée législative du Bas-Canada en 1801. Ce texte qui incorpore la Compagnie des propriétaires des eaux de Montréal décrit, dans la loi, les pouvoirs de la compagnie, ses obligations, la répartition du capital-actions et le fonctionnement du comité.

Mais, la première ouverture de l'entreprise à son environnement et sa prise en compte des stakeholders ressort de l'incorporation intitulée Président, directeur et compagnie de la Banque de Montréal. Le paragraphe 9 de l'article 9 précise la responsabilité des administrateurs dans le cas où le montant total des dettes excèderait le triple du montant du capital actuellement versé à la banque. En d'autres termes, transparaît de cette disposition une préoccupation tournée vers les créanciers qui risquent de se trouver confronter aux difficultés financières de la compagnie.

Ce sentiment se précise davantage à compter de 1840. En effet, deux lois générales (Acte pour autoriser l'établissement de Compagnies à Fonds Social dans le Bas-Canada, pour la constructions de Chemins Macadamisés, Ports et autres travaux mentionnés et Acte pour établir le libre commerce de banque en cette province, et pour d'autres fins relatives aux banques et aux affaires de banque) détaillent le régime de publicité de certains groupements. La première loi de 1849 indique que la création d'une association doit être publiée dans 
l'église de la région. La deuxième loi fait dépendre l'incorporation du dépôt et de l'enregistrement des clauses d'association au greffier de la cour de comté. Par la mise en place d'un tel dispositif, se trouve confirmé le fait que la société se tourne vers l'extérieur et doit être appréhendé par des personnes qui n'en font pas partie. Le droit facilite, par ce biais, la connaissance de l'existence de la structure sociale qui se trouve contrainte - dans une certaine mesure - à tenir compte de ses actuels et futurs partenaires.

C'est à compter de 1850 et de l'Acte pour pourvoir à la formation de compagnies incorporées à fonds social, pour des fins relatives aux manufactures, aux mines, à la mécanique ou à la chimie que les choses s'accélèrent. D'une part, cet Acte renforce la publicité dont la compagnie fait l'objet puisque la déclaration d'intention de la compagnie doit comprendre le nom corporatif, les objets de la compagnie, le nombre total des actions et leur valeur nominale. Par ailleurs, la société doit être enregistrée au bureau du Registraire du comté dans laquelle la compagnie veut faire affaire. Un duplicata est envoyé au Secrétariat provincial. Currie mentionne ainsi qu'une protection efficace du créancier est assurée par le dispositif de l'enregistrement au bureau de l'officier du comté (A. W. Currie). De plus, les fausses déclarations faites dans le Rapport Annuel des affaires entrainent la responsabilité des administrateurs, disposition qui renforce la fiabilité des informations mise en circulation à destination des partenaires de l'entreprise. D'autre part, allant au-delà même de l'information qui est diffusée, cette règlementation instaure, à la charge des actionnaires, une responsabilité des salaires des employés et une responsabilité pour les dettes de la compagnie tant que le montant total du fonds social n'a pas été payé. Au travers de ces dernières dispositions, une préoccupation en faveur des employés et des créanciers de la société ressort nettement.

Par la suite, la première loi québécoise des compagnies (Loi des compagnies) témoigne de l'appréhension par les entreprises des éléments qui l'entourent. Non seulement le sort des actionnaires se trouve amélioré par rapport à la période antérieure, mais encore les tiers sont protégés au travers d'une série de mécanismes. Une partie de la doctrine canadienne indique que l'un des objectifs de la Loi des compagnies (droit civil) est de régir les relations juridiques entre les corporations privées et leurs membres (actionnaires), leurs représentants (dirigeants, employés) ou les tiers (J. Smith et Y. Renaud). En premier lieu, les actionnaires voient leur possibilité de procéder à un contrôle interne de la firme augmentées par le biais de l'instauration d'une procédure d'inspection ${ }^{2}$, de la nomination de vérificateurs ${ }^{3}$ et de l'accès à

\footnotetext{
${ }^{2}$ Dans ses grandes lignes, le dispositif prévoyait que les déclarations exigées des compagnies qui faisaient affaires dans la province de Québec devaient lui être transmises. De même, le rapport annuel ou le prospectus requis des entreprises incorporées au Québec ou y faisant affaires était soumis au ministre.
} 
des informations sociales ${ }^{4}$. En deuxième lieu, les «tiers» d'une entreprise sont pris en considération par le droit. Tout d'abord, un souci de protection des tiers se manifeste dans le domaine des sociétés en formation. Ainsi, la jurisprudence québécoise accorde-t-elle une protection aux tiers sur les bases du Code civil ou de la Loi des compagnies. Ensuite, le droit des sociétés québécois offre aux tiers une double protection au cours du fonctionnement de l'entreprise. A ce propos, Smith note : "There are a host of principles in civil law by which protection can be afforded to third party (...) The various ways by which this can be done may be conveniently grouped under two separate headings: those relating to civil law mandate and to quasi-contracts ». Enfin, une information non négligeable est mise à la disposition de l'ensemble de ceux intéressés par l'existence et le fonctionnement d'une entreprise. Les registres et archives qui recensent des informations précieuses sur l'entreprise (déclaration exigée des compagnies faisant affaire au Québec, rapport annuel, prospectus, rapport spécial de toutes matières demandé par le ministre, lettre patentes, documents dont l'enregistrement était imposée par l'article 2 (2) de la Loi des compagnies) sont tenus à la disposition du public (article 2 (3) de la Loi des compagnies) et le ministre doit fournir des copies de ses lettres patentes ainsi que leur enregistrement et délivrer sous sa signature des certificats à cet effet à toute personne qui les demande (article 2 (4) de la Loi des compagnies). La disponibilité de cette information est assurée par un dispositif de sanction relativement lourd. La compagnie, ses administrateurs et, dans le cas d'une compagnie constituée hors la province de Québec, ses représentants dans la province sont passibles d'une amende (article 5, Loi des déclarations des compagnies et des sociétés et articles 3, 4 (4) et 5 de la Loi des renseignements sur les compagnies). Dans le cas d'omission de produire le rapport annuel ou, le rapport spécial prévus par la Loi des renseignements sur les compagnies, les compagnies incorporées en vertu d'une loi du Québec sont également passibles de dissolution (article 5 (a), (b) et (c) de la Loi des renseignements sur les compagnies et article 24 de la Loi des compagnies). Mais, la Loi des compagnies va plus loin et donne à l'Etat la possibilité d'enquêter lorsqu'un acte frauduleux ou une infraction à une règlementation relative aux entreprises a été commise ou est sur le point de l'être (article 3 de la Loi des compagnies).

\footnotetext{
${ }^{3}$ L'article 107 de la Loi des compagnies précisait que le ministre peut nommer un ou des inspecteurs pour examiner les affaires d'une compagnie et lui faire rapport, à la demande d'actionnaires dont il juge le nombre suffisant. L'article 110 (2) indiquait que les actionnaires pouvaient demander que le ministre nomme et fixe la rémunération d'un vérificateur lorsqu'aucun vérificateur n'a été nommé.

${ }^{4}$ L'ensemble du registre et des archives requis pour l'application de la Loi des compagnies étaient ouverts à l'examen du public (article 2 (3), Loi des compagnies).
} 
Enfin, l'adoption en 1985 de la Loi canadienne sur les sociétés par actions ne doit pas être négligée. En effet, résultant d'une réforme majeure initiée durant les années 1970 par le Comité Dickerson (R. W. V. Dickerson, L. Getz et J. L. Howard), cette loi se veut protectrice de cette catégorie spécifique de partenaires de l'entreprise que sont les actionnaires.

\subsection{Position contemporaine de la jurisprudence canadienne : l'acception large de l'intérêt de l'entreprise}

Le droit canadien des sociétés démontre une perméabilité des frontières de l'entreprise avec son environnement à travers la notion d'intérêt social et la manière globale dont celui-ci est perçu.

L'arrêt de la Cour suprême du Canada Magasins à rayons Peoples Inc. (Syndic de) v. Wise de 2004 (Magasins à rayons Peoples Inc. (Syndic de) v. Wise, 2004 CSB 68) a bouleversé de nombreuses idées reçues en droit des sociétés. En l'espèce, un des problèmes juridiques soumis aux magistrats de la Cour suprême était de déterminer si la mise en place d'une nouvelle politique d'approvisionnement par les administrateurs de la société Peoples constituait une transgression des devoirs de prudence, de diligence, de loyauté et de bonne foi prévus par l'article 122 de la Loi canadienne sur les sociétés par actions. Concernant strictement le devoir de loyauté, la Cour suprême procède à un revirement de jurisprudence et rompt avec sa vision traditionnelle de l'intérêt de la firme fondée sur la primauté des actionnaires et ce, en faveur d'une conception plus large qui reconnaît la pertinence des intérêts de toutes les parties prenantes (S. Rousseau). La Cour indique « (...) qu'il ne faut pas interpréter l'expression "au mieux des intérêts de la société » comme si elle signifiait simplement « au mieux des intérêts des actionnaires » ». De plus, les magistrats notent que « (...) pour déterminer s'il s'agit au mieux des intérêts de la société, il peut être légitime pour le conseil d'administration, vu l'ensemble des circonstances dans un cas donné, de tenir compte notamment des intérêts des actionnaires, des employés, des fournisseurs, des créanciers, des consommateurs, des gouvernements et de l'environnement ». Abandonnant l'ancienne domination de l'intérêt des actionnaires, les administrateurs ne peuvent plus désormais être guidés uniquement par le critère de la maximisation de la valeur pour les actionnaires et doivent chercher à maximiser la valeur de l'entreprise.

Récemment, la décision BCE Inc. v. Détenteurs de débentures de 1976 est venue confirmer l'arrêt Peoples et fournir divers enseignements complémentaires (BCE Inc. v. Détenteurs de débentures de 1976, 2008 CSC 69). Dans cette affaire, la Cour suprême du Canada relève que "[l]'obligation fiduciaire des administrateurs envers la société (...) leur impose d'agir au 
mieux des intérêts de la société. Souvent les intérêts des actionnaires et des parties intéressées concordent avec ceux de la société. Toutefois, lorsque ce n'est pas le cas, l'obligation des administrateurs est claire : elle est envers la société ». De plus, les juges indiquent que : "[l]'obligation fiduciaire des administrateurs est un concept large et contextuel. Elle ne se limite pas à la valeur des actions ou au profit à court terme. Dans le contexte de la continuité de l'entreprise, cette obligation vise les intérêts à long terme de la société. Son contenu varie selon la situation ». Enfin, la Cour suprême fait expressément référence à la notion de RSE en notant que "[l]orsque le conflit [soit entre les intérêts de différentes parties intéressées, soit entre les intérêts des parties intéressées et ceux de la société] touche les intérêts de la société, il revient aux administrateurs (...) d'agir au mieux des intérêts de la société en tant qu'entreprise socialement responsable».

\section{Etat des lieux en droit américain : vers une dilution progressive des frontières de l'entreprise}

Le droit américain des sociétés cultive l'incertitude. Bien que la tendance jurisprudentielle au dépassement de la prise en compte des actionnaires ne soit pas affirmée nettement, la «schizophrénie» de la conception légale de l'entreprise profite aux défenseurs d'une conception ouverte de l'entreprise (W. T. Allen). Le doute dont la jurisprudence est la cause est, en effet, accentué par le contenu des statuts des sociétés américaines qui renvoient au concept d' "interests of the corporation » dans l'appréciation des devoirs pesant sur les dirigeants. Près de la moitié des Etats américains ont ainsi adopté le «constituency statutes » qui permet au conseil d'administration de prendre en compte les intérêts des non actionnaires dans la prise de décision dans le meilleur intérêt de la société (I. B. Lee). Si l'obligation de tenir compte d'un certain nombre de groupes de partenaires de l'entreprise est inscrite dans les textes, elle a un effet limité (L. E. Mitchell). En premier lieu, un grand nombre de ces dispositions ne s'applique qu'en cas d'offres publiques d'achat hostiles. En deuxième lieu, toutes ces dispositions (excepté une) n'ont pas de caractère obligatoire : les administrateurs peuvent en tenir compte. Troisièmement, aucune des dispositions ne permet aux intéressés d'intenter un procès aux administrateurs pour ne pas avoir tenu compte de leurs intérêts.

\subsection{Un sentiment qui se retrouve auprès des magistrats ...}

Si le procès opposant les frères Dodge à Ford Motor en 1919 témoigne d'une conception exclusivement actionnariale de l'entreprise (Dodge v. Ford Moto Co., 170, N.W., 668, 684

(Mich. 1919)), des décisions américaines ultérieures permettent de se rendre compte de 
l'évolution de la perception juridique de l'entreprise et du terrain gagné par le développement durable, la RSE et la stakeholder theory. Rappelons que dans le fameux arrêt Dodge v. Ford Moto Co., la Cour suprême du Michigan a indiqué que les seuls ayant droits de l'entreprise étaient les actionnaires. En vertu de cette décision, l'entreprise est donc organisée d'abord pour le profit des actionnaires et la latitude discrétionnaire des dirigeants ne doit être mobilisée que dans cet unique but. L'entreprise se trouve finalement centrée sur les seuls actionnaires et tout élément extérieur lui est indifférent.

Premièrement, rompant avec cette décision, l'affaire Smith Manufacturing Co contre Barlow de 1953 redéfini le but de l'entreprise (Smith Manufacturing v. Barlow, dans Beauchamp et Bowie). En l'espèce, la Cour suprême du New Jersey soutient que : «(...) les actionnaires individuels, dont les intérêts privés reposent entièrement sur le bien-être de l'entreprise, ne devraient pas fermer leurs yeux sur les réalités présentes et contrarier l'action à long terme de l'entreprise, en reconnaissant et en s'acquittant volontairement de leur obligations ».

Deuxièmement, dans l'affaire Unocal Corp. v. Mesa Petroleum Co. de 1985 (Unocal Corp. v. Mesa Petroleum Co., 493 A.2d 946 (Del. 1985)), la Cour suprême du Delaware indique que le conseil d'administration doit considérer l'impact d'une offre publique d'achat sur les constituants d'une entreprise autres que les actionnaires (créditeurs, consommateurs, employés et peut être la communauté en général) $)^{5}$.

Troisièmement, dans le cas d'un conflit d'intérêts entre les actionnaires et les autres partenaires de l'entreprise, la Cour du Delaware a évolué au travers de sa décision Crédit Lyonnais Bank Nederland N.V. v. Pathé Communications Corp. (Crédit Lyonnais Bank Nederland N.V. v. Pathé Communications Corp., No 12150, 1991 Del. Ch. LEXIS, 215108 (Dec. 30, 1991)). Dans cette affaire où une société se retrouvait en difficultés financières, les actionnaires n'ont pu mettre en jeu la responsabilité du conseil d'administration pour avoir refusé de poursuivre une stratégie à haut-risque préférée par ces actionnaires, mais nuisibles aux intérêts des créanciers de la société.

A ce panorama jurisprudentiel esquissé dans ces grands traits, il faut ajouter que les juridictions américaines n'hésitent pas à autoriser la prise en compte par les administrateurs des actionnaires présents et futurs et laissent entrevoir une protection des créanciers (par exemple : Re Trizec Corp., [1994] 10 W.W.R. 127 : Atla. C.A.).

\footnotetext{
${ }^{5}$ Le domaine des offres publiques témoigne d'un mouvement de balancier de la jurisprudence, bien qu'une évolution soit cependant perceptible. En effet, suite à l'affaire Unocal v. Mesa Petroleum, diverses décisions judiciaires ont fait dépendre la prise en compte des non actionnaires de la présence de bénéfices revenant aux actionnaires et ont fait prévaloir l'obtention du meilleur prix pour les actionnaires (Paramount Communications, Inc. v. QVC Network Inc., 637 A.2d 34 (Del. 1994) ; Paramount Communications, Inc. v. Time, Inc., 571 A.2d 1140 (Del. 1989) ; Revlon v. MacAndrews, 506 A.2d 173, 193 (Del. 1986)).
} 


\section{2. ... Qui se confirme auprès des auteurs américains}

Un détour par la doctrine juridique américaine démontre qu'une place est faite à d'autres partenaires que les actionnaires. Une partie des auteurs nord-américains adresse des critiques sévères à la centralisation du droit des sociétés sur le seul intérêt des actionnaires (en plus des auteurs cités : J. Mahoney, C. C. Asher and J. Mahoney ; L. A. Stout ; L. E. Strine ; M. M. Blair and L. A. Stout). Deux auteurs sont éclairants sur ce point. D'une part, Stout conteste l'assimilation de l'objectif de la firme à l'intérêt des actionnaires en mettant en avant l'insuffisance des assises théoriques fondant cette opinion (L. A. Stout). D'autre part, Greenfield développe de nouveaux principes pour le droit américain des sociétés dont l'élément central est que les entreprises doivent servir les intérêts de la société «as a whole » (K. Greenfield).

D'autres auteurs américains bâtissent des ponts entre théorie des parties prenantes et monde juridique, et ce bien qu'ils se montrent plus réservés. Ainsi, Hansmann et Kraakmann écrivent qu'un des rôles de la loi est de solutionner le problème d'agence identifié entre l'entreprise et les créditeurs, les employés et les consommateurs avec lesquelles celle-ci a contracté ( $\mathrm{H}$. Hansmann and R. Kraakman). Bien qu'il se montre dubitatif sur le sort réservé à une telle attitude, Mitchell souligne qu'il est de l'intérêt des sociétés anonymes américaines de tenir compte d'autres partenaires que les actionnaires (L. E. Mitchell). Proche, Blair souligne que la loi donne le sentiment dans corpus que les entreprises ont un but social qui s'ajoute à celui de fournir des profits aux actionnaires (M. M. Blair).

\section{Conclusion : une entreprise sans frontières qui implique une redéfinition de sa valeur}

L'ouverture de l'entreprise aux nouveaux paradigmes que sont le développement durable, la RSE ou encore, la théorie des parties prenantes est confirmée. Le paysage qui se dessine au plan juridique confirme le retour, au sein des sciences sociales, de l'acteur et du sujet mais obscurcissent dans le même temps les frontières de l'entreprise. Un modèle d'organisation industrielle nouveau tend à se développer dans lequel les stratégies coopératives entre une variété d'agents fortement interdépendants redéfinissent l'entreprise (M.-H. Depret et A. Hamdouch). Cette dernière apparaît comme une constellation d'intérêts collectifs ou un jeu de contrats multilatéraux entre stakeholders (S. M. Bainbridge ; R. E. Freeman and W. N. Evan) dont l'importance et les attentes sont variables, mais qui sont unis dans un but particulier (S. Mercier).

Aux images traditionnelles de l'entreprise, objet des actionnaires insensible à toute autre influence, se substitue un paysage nouveau dans lequel les règles ont pour objectif de mettre 
de l'ordre dans l'enchevêtrement des liens et des réseaux. Entre tous les acteurs, le droit participe à la définition des règles du jeu, des systèmes institutionnels et des mécanismes permettant de résoudre les conflits non prévus dans les contrats initiaux passés avec eux (O. Hart). Ainsi, dans les sociétés dont le capital est représenté en tout ou partie par des actions anonymes et librement cessibles sur le marché financier, le droit - instrument générique de règlement des conflits - permet de définir, délimiter et défendre les droits des multiples stakeholders.

Cette conclusion sur le terrain juridique conforte les analyses d'auteurs tels que Post, Preston et Sachs (J. Post, L. Preston and S. Sachs), Chandler (A. D. Chandler), Aoki (M. Aoki (a) ; M. Aoki $(b))$, Coriat (B. Coriat), Aglietta (M. Aglietta), Savall et Zardet (H. Savall et V. Zardet) ou encore, Pesqueux (Y. Pesqueux. Voir aussi : Y. Pesqueux et B. Triboulois). Cette vision nouvelle de l'entreprise consacrant un élargissement de l'éventail de ses parties prenantes s'inscrit ergo propter hoc dans la remise en cause de la valeur de l'entreprise qui se retrouve présente dans de nombreux écrits financiers. De l'étude de la littérature actuelle en ce domaine, il faut encore avoir recours à la performance financière d'une entreprise pour savoir si les attentes de ses stakeholders sont respectées et, inversement, qu'il faut mesurer la performance sociale pour déterminer la performance financière (J. Igalens et J.-P. Gond). Or, la logique qui fonde le système comptable repose prima facie sur une perception selon laquelle une société est la propriété de ses actionnaires, lesquels sont les seuls à avoir le droit de s'approprier le résidu supposé égal au profit comptable. Si comparer la performance sociale et la performance financière fournit indiscutablement des enseignements, ces deux notions sont de nature ou, d'espèce, différente. La performance financière se résume en chiffre, tient principalement compte des informations comptables et boursières et offre une vision à court-terme (M. M. Blair). La performance sociale ou la «stakeholder satisfaction » renvoie à des attentes certes monétaires mais à bien d'autres (protection dans les conditions de travail, importance de l'environnement) et implique une maximisation de la valeur de l'entreprise à long-terme (M. C. Jensen). En conséquence, l'évaluation de la « stakeholder satisfaction » doit se faire au travers d'un double critère : l'accroissement de la valeur monétaire et les autres fins de la firme (Institut Montaigne ; J. S. Harrison and R. E. Freeman ; G. Charreaux et P. Desbrières). Pour une telle mesure, l'existence d'un critère de performance quantitatif est un outil indispensable. Cependant, il faut ajouter à ce premier critère tiré de la valeur quantitative, un deuxième critère qui peut se résumer en ces termes : "l'entreprise existe pour d'autres fins que l'accroissement de sa valeur financière » (M.-H. Depret et A. Hamdouch ; L. E. Mitchell). La mesure de la performance des entreprises requiert de prendre 
en compte à la fois la diversité et l'interdépendance de l'ensemble des stakeholders de l'entreprise, aussi bien en interne, au sein de réseau interentreprises, qu'en dehors (J. Pasquero). Cette proposition se justifie d'autant plus que l'origine et la formation du revenu de l'entreprise soulèvent de délicates questions et que la notion même de performance de l'entreprise prête à discussion. D'un côté, le parcours amenant des capitaux investis dans des ressources-actifs à la création de valeur demeure complexe et implique une prise en compte de facteurs extérieurs à l'actionnariat (Y. Biondi). D'un autre côté, à l'instar de ce que relèvent les auteurs, l'accroissement de la valeur de l'entreprise sur le long terme n'est-il pas le seul outil pertinent pour mesurer réellement la performance d'une entreprise ?

\section{Bibliographie}

Aglietta Michel. Régulation et crise du capitalisme. Paris, France : Calmann-Lévy, 1982, 486 p.

Alchian Armen Albert, Demsetz, Harold. Production. Information Costs, and Economic Organization. Economic Review, 1972, vol. 62, p.777-795.

Allen William. T. Our Shizophrenic Conception of the Corporation. Cardozo Law Review, 1982, vol. 14, n² 2, p.261-282.

Aoki Masahiko (a). Towards an Economic Theory of the Japanese Firm. Journal of Economic Litterature, 1990, vol. 26, p.1-27.

Aoki Masahiko (b). Information, Incentive and Bargaining structure in the Japanese economy. Cambridge and New York, United-States : Cambridge University Press, 1988, 332 p.

Bainbridge Stephen M. Corporation Law and Economics. New York, United-States : Foundation Press, 2002, 884 p.

Biondi Yuri. La valorisation des actifs dans le cadre conceptuel de la future normalisation comptable internationale, particulièrement au regard des normes 36 et 38. Comptabilité Contrôle - Audit, 2004, T. 10, vol. 2, p.55-72.

Blair Margareth M., Stout Lynn A. Director Accountability and the Mediating Role of the Corporate Board. Washington University Law Quarterly, 2001, vol. 79, n 2, p.403-448.

Blair Margareth M. Ownership and control : Rethinking corporate governance for the twentyfirst century. Washington D.C., United-States : The Brookings Institution, 2005, 371 p. Brunhes Bernard. Réflexions sur la gouvernance. Droit Social, 2002, n² 2, p.115-120. 
Capron Michel, Quairel-Lanoizelée Françoise. La responsabilité sociale d'entreprise. Paris, France : La Découverte, 2007, 314 p.

Capron Michel, Quairel-Lanoizelée Françoise. Mythes et réalités de l'entreprise responsable : Acteurs - Enjeux - Stratégies. Paris, France : La Découverte, 2004, 252 p.

Chandler Adolph D. Organizational capabilities and the Economic History of the Industrial Enterprise. Journal of Economic Perspectives, 1992, vol. 6, $\mathrm{n}^{\circ} 3$.

Charreaux Gérard (a). La théorie positive de l'agence : positionnement et apports. Cahier du FARGO, décembre 1999, http://ungaro.u-bourgogne.fr/WP/0991201.pdf.

Charreaux Gérard (b). La théorie positive de l'agence : lecture et relectures ... Cahier du FARGO, septembre 1998, http://ungaro.u-bourgogne.fr/WP/0980901.pdf.

Charreaux Gérard, Desbrières Philippe. Gouvernance des entreprises : valeur partenariale contre valeur actionnariale. Finance Contrôle Stratégie, 1998, vol. 1, n² 2, p.57-88.

Coriat Benjamin. Science, Technique et Capital. Paris, France : du Seuil, 1981, 186 p.

Currie Arthur W. The First Dominion Companies Act. Canadian Journal of Economics and Political Science, 1962, vol. 28, p.387-404.

Depret Marc-Hubert, Hamdouch Abdelillah. Gouvernement d'entreprise et performance. Dans Gouvernement d'entreprise : Enjeux managériaux, comptables et financiers, Alain Finet (dir.), Bruxelles, Belgique : De Boeck., 2005, 269 p., p.39-80.

Despax Michel. L'entreprise et le droit. Paris, France : L.G.D.J., 1956, 443 p.

Dickerson Robert W. V., Getz Leon et Howard John L. Propositions pour un nouveau droit des corporations commerciales canadiennes, 2 vol., Ottawa, Canada : Information Canada, 1971.

Freeman R. Edward and al. Managing for Stakeholders: Survival, Reputation, and Success. United-States : Yale University Press, 2007, 284 p.

Freeman R. Edward, Evan William N. Corporate Governance : A Stakeholder Interpretation. The Journal of Behaviour Economics, 1990, vol. 19, $\mathrm{n}^{\circ}$ 4, p.337-359.

Greenfield Kent. The Failure of Corporate Law. Chicago, United-States : The University of Chicago Press, 2006, 257 p.

Hansmann Henry, Kraakman Reinier. Agency Problems and Legal Strategies. Dans The Anatomy of Corporate Law : A comparative and Functional Approach, Reinier Kraakman et al. (dir.), Oxford, United-States : Oxford University Press, 2004, 231 p., p.21-32.

Harrison J. S., Freeman R. Edward. Stakeholders, Social Responsibility, and Performance: Empirical Evidence and Theoretical Perspectives. Academy of Management Journal, 1999, vol. $42, \mathrm{n}^{\circ} 5$, p.479-486. 
Hart Oliver. Corporate Governance : Some Theory and Implications. Economic Journal, vol. $105, \mathrm{n}^{\circ} 430, \mathrm{p} .678-689$.

Igalens Jacques, Gond Jean-Pascal. La mesure de la performance sociale de l'entreprise : une analyse critique et empirique des données ARESE. Revue de Gestion des Ressources Humaines, 2003, $\mathrm{n}^{\circ}$ 50, p.111-148.

Ionescu-Somers Aileen. View of Managers about their Stakeholder Environment : An Update. Dans Inside the Mind of the Stakeholder: The Hype Behind Stakeholder Pressure, Ulrich Steger (dir.), Bruxelles, Belgique : IMD International, EABIS, European Academy of business in society, 2007, 318 p., p.29-41.

Institut Montaigne. Mieux gouverner l'entreprise. mars 2003, 57 p.

Jensen Michaël C. Value Maximisation, Stakeholder Theory, and the Corporate Objective Function. European Financial Management, 2001, vol. 7, n 3, p.297-317.

Jensen Michaël C., Meckling William H., Theory of the Firm : Managerial Behaviour. Agency Costs and Ownership Structure, Journal of Financial Economics, 1976, vol. 3, p.305357.

Lee Ian Bee. Corporate Law, Profit Maximization, and the "Responsible "Shareholder. Stanford Law Business and Financial, 2004-2005, vol. 10, n 31, p.31-62.

Lozano Joseph M., Albareda Laura, Ysa Tamyko. Governments and corporate social responsibility Public policies beyond regulation and voluntary compliance. ESADE, Palgrave MacMillan, 2007, 208 p.

Lucas François-Xavier. Développement durable et droit des sociétés ... Bulletin Joly Sociétés, 2008, n 4, p.267.

Maati Jérôme. Le gouvernement d'entreprise. Bruxelles, Belgique : de Boeck Université, 1999, 293 p.

Mahoney James, Asher Carleton C. and Mahoney Jospeh. Towards a Property Rights Foundation for a Stakeholder Theory of the Firm. Journal of Management and Governance, 2005, vol. 9, $\mathrm{n}^{\circ} 1$, p.5-32.

Martel Maurice, Martel Paul. La compagnie au Québec, Vol. 1, Canada: éd. Wilson \& Lafleur, Martel Itée, 2008, 1800 p.

Mercier Samuel. L'apport de la théorie des parties prenantes au management stratégique : une synthèse de la littérature. XIème Conférence de l'Association Internationale de Management Stratégique, juin 2001, http://www.strategie-aims.com/quebec/web/actes/f-152cd.pdf. 
Mitchell Lawrence E. La Firme irresponsable ..., traduit de l'américain par P. Rimbault, Paris, France : Economica, 2003, 262 p.

Nick Alexander. Financial Institutions : Corporate Sustainability as a Significant Niche?. Dans Inside the Mind of the Stakeholder: The Hype Behind Stakeholder Pressure, Ulrich Steger (dir.), Bruxelles, Belgique : IMD International, EABIS, European Academy of business in society, 2007, 318 p., p.131-156.

Paillusseau Jean. Entreprise, société, actionnaires, salariés, quels rapports ?. Recueil Dalloz, 1999, chron., p.157-166.

Pasquero Jean. La responsabilité sociale de l'entreprise comme objet des sciences de gestion : regard historique. Dans Responsabilité sociale et environnementale de l'entreprise, MarieFrance Turcotte et Anne Salmon (dir.), Sainte-Foy, Canada: Presses de l'Université du Québec, 2005, 228 p., p.80-111.

Pesqueux Yvon, Triboulois Bruno. La dérive organisationnelle : Peut-on encore conduire le changement? Paris, France : L'Harmattan, 2004, 221 p.

Pesqueux Yvon. L'entreprise mise à nu. Paris, France : Economica, 1987, 266 p.

Post James, Preston Lee, Sachs Sybille. Redefining the Corporation : Stakeholder Management and Organizational Wealth. Stanford, United-States : Stanford University Press, 2002, 376 p.

Quélin Bertrand. Les frontières de la firme. Paris, France : Economica, 2002, 138 p.

Ripert Georges. Aspects juridiques du capitalisme moderne. Paris, France : L.G.D.J., 1998 ( $2^{\text {ème }}$ éd.), $354 \mathrm{p}$.

Roselle James, The triple Bottom Line: Building Shareholder Value. Dans Corporate Responsibility : The Corporate Governance of the $21^{\text {st }}$ Century, Ramon Mullerat (dir.), International Bar Association Series, 2005, 596 p., p.113-141.

Rousseau Stéphane. Sans frontières? Les devoirs des administrateurs de sociétés par actions à la lumière de la jurisprudence récente. Cours de perfectionnement du notariat, 2006, $\mathrm{n}^{\circ} 1$, p.101-157.

Savall Henry, Zardet Véronique. Tétranormalisation : Défis et dynamiques, Paris, France : Economica, 2005, 195 p.

Smith John, Renaud Yves. Droit québécois des corporations commerciales, Vol. I, Judico, 1974.

Stout Lynn A. Why we Should Stop Teaching Dodge v. Ford ?. Law \& Economic Research Paper Series, Research Paper n07-11. 
Strine Leon E. The Social Responsibility of Boards of Directors and Stockholders in Change of Control Transactions : Is There Ane «There » There ?. California Law Review, 2002, vol. 75, p.1169-1188.

Tchotourian Ivan (a). La responsabilité sociale des entreprises ou quand le monde des entreprises change. Revue LexElectronica, Hiver 2009, vol. 13, n 13, p.1-53.

Tchotourian Ivan (b). «Informing Good To Do Well»: Une approche comparée de la participation (imparfaite) du droit des sociétés par actions au management responsable. La Revue des Sciences de gestion, septembre-octobre 2008, nº 233, p.35-42.

Torres Félix. Une idée neuve à réinventer, l'entreprise. Dans Repenser l'entreprise : : Saisir ce qui commence, vingt regards sur une idée neuve, Jacques Chaize et Félix Torres (dir.), Paris, France : le cherche midi, 2008, 348 p., p.17-24.

Trébulle François-Guy (a). Le développement de la prise en compte des préoccupations environnementales, sociales et de gouvernance. Droit des sociétés, 2009, étude 1, p.7-11.

Trébulle François-Guy (b). Stakeholder theory et droit des sociétés. Bulletin Joly Sociétés, 2007, n 1, p.7-32.

Trébulle François-Guy (b). Stakeholders Theory et droit des sociétés. Bulletin Joly Sociétés, 2006, nº 123, p.1337-1354. 